Volume 133, Number 1, Pages 275-283

S 0002-9939(04)07501-X

Article electronically published on July 26, 2004

\title{
ALGEBRAIC POLYNOMIALS WITH NON-IDENTICAL RANDOM COEFFICIENTS
}

\author{
K. FARAHMAND AND A. NEZAKATI
}

(Communicated by Richard C. Bradley)

\begin{abstract}
There are many known asymptotic estimates for the expected number of real zeros of a random algebraic polynomial $a_{0}+a_{1} x+a_{2} x^{2}+$ $\cdots+a_{n-1} x^{n-1}$. The coefficients $a_{j}(j=0,1,2, \ldots, n-1)$ are mostly assumed to be independent identical normal random variables with mean zero and variance unity. In this case, for all $n$ sufficiently large, the above expected value is shown to be $O(\log n)$. Also, it is known that if the $a_{j}$ have non-identical variance $\left(\begin{array}{c}n-1 \\ j\end{array}\right)$, then the expected number of real zeros increases to $O(\sqrt{n})$. It is, therefore, natural to assume that for other classes of distributions of the coefficients in which the variance of the coefficients is picked at the middle term, we would also expect a greater number of zeros than $O(\log n)$. In this work for two different choices of variance for the coefficients we show that this is not the case. Although we show asymptotically that there is some increase in the number of real zeros, they still remain $O(\log n)$. In fact, so far the case of $\operatorname{var}\left(a_{j}\right)=\left(\begin{array}{c}n-1 \\ j\end{array}\right)$ is the only case that can significantly increase the expected number of real zeros.
\end{abstract}

\section{INTRODUCTION}

Let $N_{n}(a, b)$ be the number of real zeros of a random algebraic polynomial

$$
P_{n}(x)=\sum_{j=0}^{n-1} a_{j} x^{j},
$$

in the interval $(a, b)$ where the coefficients $\left\{a_{j}\right\}_{j=0}^{n-1}$ are a sequence of random variables. For the case of normal standard distribution of the coefficients, it is known that the mathematical expectation of $N_{n}(-\infty, \infty)$ is asymptotic to $(2 / \pi) \log n$ as $n \rightarrow \infty$. This asymptotic value remains valid for the general case when the coefficients belong to the domain of attraction of the normal law. For those distributions that do not belong to the above domain, Logan and Shepp [9] and [10] show there is a slight increase to the coefficient $(2 / \pi)$ obtained for $E N_{n}(-\infty, \infty)$. Wilkins [14] obtained an interesting result by showing that the error term for the asymptotic

Received by the editors February 18, 2003 and, in revised form, September 8, 2003.

2000 Mathematics Subject Classification. Primary 60G99; Secondary 60H99.

Key words and phrases. Number of real zeros, real roots, random algebraic polynomials, KacRice formula, non-identical random variables.

This work was completed while the second author was visiting the Department of Mathematics at the University of Ulster. The hospitality of the Department of Mathematics at the University of Ulster is appreciated. The financial support for this visit was supported by the Ministry of Science, Research and Technology of I. R. Iran. 
formula is small and is in fact independent of $n$. Other interesting results in this direction are due to Sambandham [12] and [13] and Farahmand and Hannigan [5] and [6]. These results and other related topics are discussed in some detail in two books, one by Bharucha-Reid and Sambandham [1], the other by Farahmand [3].

It was assumed that for all classes of distributions for the coefficients in (1.1), $E N_{n}(-\infty, \infty)$ remains asymptotically $O(\log n)$. However, in an interesting development, Edelman and Kostlan 2, among other results, show that if the variance of $a_{j}$ is $\left(\begin{array}{c}n-1 \\ j\end{array}\right)$, then there is a significant increase to the expected number of real zeros. They show, for all $n$, that $E N_{n}(\infty, \infty)=\sqrt{n-1}$. It is also shown in 4 that this result is persistent for the other properties of $P_{n}(x)$, such as the expected number of maxima and minima and the expected number of times that $P_{n}(x)$ assumes a constant value $K$.

As it is characteristic of $\left(\begin{array}{c}n-1 \\ j\end{array}\right), j=0,1, \ldots, n-1$, to be maximized at the middle term for $j=[n / 2]$, so it is natural to be tempted to conjecture that for other classes of distributions with the same above property, $E N_{n}(-\infty, \infty)=O(\sqrt{n})$; see also 7]. To this end in this paper we consider two cases, both of which satisfy the above assumption. However, our theorems surprisingly show that $E N_{n}(-\infty, \infty)$ remains $O(\log n)$. It is interesting to note that with the assumption of our Theorem 1 , the rate of increase of variances is significantly larger than the case when $\operatorname{var}\left(a_{j}\right)=$ $\left(\begin{array}{c}n-1 \\ j\end{array}\right)$. Still, $E N_{n}(-\infty, \infty)$ remains significantly lower than $O(\sqrt{n})$, although it is increased from $(2 / \pi) \log n$, the case of identical variance. Therefore $\operatorname{var}\left(a_{j}\right)=$ $\left(\begin{array}{c}n-1 \\ j\end{array}\right)$ so far is the only known case in which the expected number of zeros of $P_{n}(x)$ significantly increases. It is interesting to ask if it is also unique. The main distinctive property that these authors could find for the case of $\left(\begin{array}{c}n-1 \\ j\end{array}\right)$ is that for this case the zeros are not clustered around any points in the interval $(-\infty, \infty)$. While for the case we study here, as well as the case of identical variances, the zeros are concentrated around some points. There should be other assumptions on the distribution of the coefficients that would lead to this characteristic for the zeros and would therefore increase the number of zeros. Hence, the non-identical distributed coefficients are of special interest. To this end for any constant $\sigma>1$ such that $\sigma \nrightarrow 1$ as $n \rightarrow \infty$ we prove:

Theorem 1. If the random variables $a_{j}, j=0,1,2, \ldots, n-1$, are normally distributed with mean zero and variances $\sigma^{j}$ for $0 \leq j \leq m-1$ and $\sigma^{n-j}$ for $m \leq j \leq n-1$ where $m=[n / 2]+1$, then for all sufficiently large $n$, the expected number of real zeros of $P_{n}(x)$ in (1.1) is

$$
E N_{n}(-\infty, \infty) \sim\left(\frac{4}{\pi}\right) \log n
$$

Theorem 2. With the same assumptions as in Theorem 1 with $\operatorname{var}\left(a_{j}\right)=j(n-j)$ for $0 \leq j \leq n-1$, we have

$$
E N_{n}(-\infty, \infty) \sim\left(\frac{2 \sqrt{2}}{\pi}\right) \log n .
$$

For the proof of the theorems we use an approach based on Kac's [8] or Rice's 11] results. In this case, for

$$
\begin{aligned}
A^{2} & =\operatorname{var}\left(P_{n}(x)\right), & & B^{2}=\operatorname{var}\left(P_{n}^{\prime}(x)\right), \\
C & =\operatorname{cov}\left(P_{n}(x) P_{n}^{\prime}(x)\right), & \text { and } & \Delta^{2}=A^{2} B^{2}-C^{2},
\end{aligned}
$$


the expected number of real zeros is given by the Kac-Rice formula as

$$
E N_{n}(a, b)=\frac{1}{\pi} \int_{a}^{b} \frac{\Delta}{A^{2}} d x .
$$

In order to use (1.3) to obtain $E N_{n}(-\infty, \infty)$, we note that changing $x$ to $1 / x$ and $x$ to $-x$ leaves the distribution of the coefficients of $P_{n}(x)$ in (1.1) invariant. Hence the expected number of real zeros in the interval $(0,1)$ is asymptotically the same as in $(1, \infty),(-1,0)$ and $(-\infty,-1)$. Therefore it suffices to give the result for $E N_{n}(0,1)$ only. To this end, in the following section, we present our calculations for any $m$; see, for example, (2.4)-(2.6) below. However, in order for our result to remain valid we only need a special case of $m$ given in Theorem 1 . It seems our method could also be used for the case of any $m=[n / 2]+O(1)$. However, the gain in considering this case does not justify the complicated analysis in the calculation.

\section{Proof of TheOREM 1}

From the assumptions of Theorem 1 for the distributions of the coefficients of $P_{n}(x)$, from (1.2) we can easily show that

$$
\begin{aligned}
& A^{2}=\sum_{j=0}^{m-1} \sigma^{j} x^{2 j}+\sigma^{n} \sum_{j=m}^{n-1} \sigma^{-j} x^{2 j}, \\
& B^{2}=\sum_{j=0}^{m-1} j^{2} \sigma^{j} x^{2 j-2}+\sigma^{n} \sum_{j=m}^{n-1} j^{2} \sigma^{-j} x^{2 j-2},
\end{aligned}
$$

and

$$
C=\sum_{j=0}^{m-1} j \sigma^{j} x^{2 j-1}+\sigma^{n} \sum_{j=m}^{n-1} j \sigma^{-j} x^{2 j-1} .
$$

We obtain the value of the above identities for any $\sigma$ and $m$. In each case, consequent differentiation of the previous formula multiplied by $x$ yields

$$
\begin{aligned}
& A^{2}=\frac{1-\sigma^{m} x^{2 m}}{1-\sigma x^{2}}+\frac{\sigma^{n-m+1} x^{2 m}-\sigma x^{2 n}}{\sigma-x^{2}} \\
& B^{2}= \\
& \frac{\sigma+\sigma^{2} x^{2}+\left(2 m^{2}-2 m-1\right) \sigma^{m+1} x^{2 m}-(m-1)^{2} \sigma^{m+2} x^{2 m+2}-m^{2} \sigma^{m} x^{2 m-2}}{\left(1-\sigma x^{2}\right)^{3}} \\
& +\frac{m^{2} \sigma^{n-m+3} x^{2 m-2}-\left(2 m^{2}-2 m-1\right) \sigma^{n-m+2} x^{2 m}+(m-1)^{2} \sigma^{n-m+1} x^{2 m+2}}{\left(\sigma-x^{2}\right)^{3}} \\
& +\frac{\left(2 n^{2}-2 n-1\right) \sigma^{2} x^{2 n}-n^{2} \sigma^{3} x^{2 n-2}-(n-1)^{2} \sigma x^{2 n+2}}{\left(\sigma-x^{2}\right)^{3}}
\end{aligned}
$$

and

$$
\begin{aligned}
& \text { (2.6) } C=\frac{\sigma x+(m-1) \sigma^{m+1} x^{2 m+1}-m \sigma^{m} x^{2 m-1}}{\left(1-\sigma x^{2}\right)^{2}} \\
& \quad+\frac{m \sigma^{n-m+2} x^{2 m-1}-(m-1) \sigma^{n-m+1} x^{2 m+1}-n \sigma^{2} x^{2 n-1}+(n-1) \sigma x^{2 n+1}}{\left(\sigma-x^{2}\right)^{2}} .
\end{aligned}
$$


Also from (2.4), (2.5) and (2.6), for any $\sigma$ and $m$, we obtain

$$
\begin{aligned}
& \text { (2.7) } \Delta^{2}=\frac{\sigma\left(1-\sigma^{m} x^{2 m}\right)^{2}-m^{2} \sigma^{m} x^{2 m-2}\left(1-\sigma x^{2}\right)^{2}}{\left(1-\sigma x^{2}\right)^{4}} \\
& +\frac{\sigma\left(\sigma^{n-m+1} x^{2 m}-\sigma x^{2 n}\right)^{2}-(n-m)^{2} \sigma^{n-m+2} x^{2 n+2 m-2}\left(\sigma-x^{2}\right)^{2}}{\left(\sigma-x^{2}\right)^{4}} \\
& +\left(\frac{\sigma^{n-m+1} x^{2 m}-\sigma x^{2 n}+\sigma^{m+1} x^{2 n+2 m}-\sigma^{n+1} x^{4 m}}{\left(1-\sigma x^{2}\right)^{3}\left(\sigma-x^{2}\right)^{3}}\right) \\
& +\left(\frac{2 m\left(\sigma^{m+1} x^{2 n+2 m}-\sigma^{n-m+1} x^{2 m}\right)+2 n\left(\sigma^{m+1} x^{2 n+2 m}-\sigma x^{2 n}\right)}{\left(1-\sigma x^{2}\right)^{3}\left(\sigma-x^{2}\right)^{3}}\right) \\
& \quad \times\left[\left(\sigma-x^{2}\right)-\sigma^{3}\left(1-\sigma x^{2}\right)+\sigma x^{4}\left(1-\sigma^{2}\right)\right] \\
& +\frac{m^{2}\left(\sigma^{m+1} x^{2 n+2 m-2}-\sigma^{n-m+1} x^{2 m-2}\right)+n^{2}\left(\sigma^{n-m+1} x^{2 m-2}-\sigma x^{2 n-2}\right)}{\left(1-\sigma x^{2}\right)\left(\sigma-x^{2}\right)} \\
& +\frac{2 m n \sigma^{m+1} x^{2 n+2 m-2}}{\left(1-\sigma x^{2}\right)\left(\sigma-x^{2}\right)} .
\end{aligned}
$$

In order to continue the proof of Theorem 1 , we first assume that $n$ is odd. In this case, $m=n / 2+1 / 2$, and we first consider the interval $(0,(1-\epsilon) / \sqrt{\sigma})$, where for

$$
a=1-\log \log n^{10} / \log n,
$$

we let $\epsilon=n^{-a}$. In this interval, for sufficiently large $n$, we can easily show that

$$
x^{n} \leq \sigma^{-n / 2} n^{-10} \text { and } \sigma^{m / 2} x^{m} \leq n^{-5} .
$$

Also, note that $a \rightarrow 1$ as $n \rightarrow \infty$. This is necessary to obtain the result later. Now from (2.4) and (2.7), we have

and

$$
\Delta^{2}=\frac{\sigma}{\left(1-\sigma x^{2}\right)^{4}}\left\{1+O\left(n^{-10}\right)\right\}
$$

$$
A^{2}=\frac{1}{1-\sigma x^{2}}\left\{1+O\left(n^{-10}\right)\right\} \text {. }
$$

Therefore from (1.3), we can show that

$$
E N_{n}\left(0, \frac{1-\epsilon}{\sqrt{\sigma}}\right) \sim \frac{1}{\pi} \int_{0}^{(1-\epsilon) / \sqrt{\sigma}} \frac{\sqrt{\sigma}}{1-\sigma x^{2}} d x=\frac{a}{2 \pi} \log n+\frac{1}{2 \pi} \log (2-\epsilon) .
$$

Hence for all sufficiently large $n$,

$$
\operatorname{EN}_{n}\left(0, \frac{1-\epsilon}{\sqrt{\sigma}}\right) \sim \frac{1}{2 \pi} \log n
$$

Now we assume that $(1-\epsilon) / \sqrt{\sigma} \leq x \leq(1-\delta) / \sqrt{\sigma}$, where

$$
\delta=\frac{\log \log n}{n} .
$$

In this case we have

$$
\frac{1-\sigma^{m} x^{2 m}}{1-\sigma x^{2}} \leq m .
$$


Therefore, from (2.4) and (2.7), we have

$$
\Delta^{2} \leq \frac{\left(1-\sigma^{m} x^{2 m}\right)^{2}}{\left(1-\sigma x^{2}\right)^{4}}\left(1-\left(\sigma x^{2}\right)^{m-1}\right)\left\{1+O\left(\frac{(\log \log n)^{3}}{n}\right)\right\},
$$

and also

$$
A^{2} \geq \frac{1-\sigma^{m} x^{2 m}}{1-\sigma x^{2}} .
$$

Now, by using the same method as in [3, p. 33] and from (1.3), we can show that

$$
E N_{n}\left(\frac{1-\epsilon}{\sqrt{\sigma}}, \frac{1-\delta}{\sqrt{\sigma}}\right)=O(\sqrt{\log n}) .
$$

In order to consider the interval $(1-\delta) / \sqrt{\sigma} \leq x \leq(1+\delta) / \sqrt{\sigma}$, it is easy to see that, for any $x$, the integrand of (1.3) does not exceed

$$
\frac{\Delta}{A^{2}} \leq\left(\frac{B^{2}}{A^{2}}\right)^{1 / 2} \leq n
$$

and thus

$$
E N_{n}\left(\frac{1-\delta}{\sqrt{\sigma}}, \frac{1+\delta}{\sqrt{\sigma}}\right)=O(\log \log n) .
$$

When $(1+\delta) / \sqrt{\sigma} \leq x \leq(1+\epsilon) / \sqrt{\sigma}$, since

$$
\Delta^{2} \leq \sigma^{2 m} x^{4 m}\left[\frac{\left(1-\sigma^{-m} x^{-2 m}\right)^{2}}{\left(\sigma x^{2}-1\right)^{4}}\left(1-\left(\sigma x^{2}\right)^{-m-1}\right)\left\{1+O\left(\frac{(\log \log n)^{3}}{n}\right)\right\}\right]
$$

and

$$
A^{2} \geq \sigma^{m} x^{2 m}\left[\frac{1-\sigma^{-m} x^{-2 m}}{\sigma x^{2}-1}\right],
$$

similar to (2.11), from (1.3), we can show that

$$
E N_{n}\left(\frac{1+\delta}{\sqrt{\sigma}}, \frac{1+\epsilon}{\sqrt{\sigma}}\right)=O(\sqrt{\log n}) .
$$

Now we let $(1+\epsilon) / \sqrt{\sigma} \leq x \leq(1+\eta) / \sqrt{\sigma}$, where

$$
\eta=\exp \left(-(\log n)^{1 / 3}\right) .
$$

For this interval, from (2.4) and (2.7), we obtain

$$
\begin{aligned}
\Delta^{2}= & \sigma^{2 m} x^{4 m}\left(\frac{\sigma}{\left(\sigma x^{2}-1\right)^{4}}\right)\left[1+\left(\frac{\sigma x^{2}-1}{\sigma-x^{2}}\right)^{4}\right. \\
& \left.+\frac{\left(\sigma\left(\sigma-x^{2}\right)^{2}+\sigma\left(1-\sigma x^{2}\right)^{2}+x^{2}\left(1-\sigma^{2}\right)^{2}\right)\left(\sigma x^{2}-1\right)}{\sigma\left(\sigma-x^{2}\right)^{3}}+O\left(n^{-10}\right)\right]
\end{aligned}
$$

and

$$
A^{2}=\sigma^{m} x^{2 m}\left(\frac{1}{\sigma x^{2}-1}\right)\left[1+\frac{\sigma x^{2}-1}{\sigma-x^{2}}+O\left(n^{-10}\right)\right] .
$$

It is easy from (2.16) and (2.17) for this range of $x$ to show that

$$
\Delta^{2}=\sigma^{2 m} x^{4 m}\left(\frac{\sigma}{\left(\sigma x^{2}-1\right)^{4}}\right)\left[1+O\left(\eta^{2}\right)\right]
$$


and

$$
A^{2}=\sigma^{m} x^{2 m}\left(\frac{1}{\sigma x^{2}-1}\right)[1+O(\epsilon)] .
$$

Therefore, (1.3) now gives

$$
\begin{aligned}
E N_{n}\left(\frac{1+\epsilon}{\sqrt{\sigma}},\right. & \left.\frac{1+\eta}{\sqrt{\sigma}}\right) \sim \frac{1}{\pi} \int_{(1+\epsilon) / \sqrt{\sigma}}^{(1+\eta) / \sqrt{\sigma}} \frac{\sqrt{\sigma}}{\sigma x^{2}-1} d x \\
& =\left(\frac{a}{2 \pi}\right) \log n+\left(\frac{1}{2 \pi}\right) \log \left(\frac{2+\epsilon}{2+\eta}\right)-\left(\frac{1}{2 \pi}\right)(\log n)^{1 / 3} .
\end{aligned}
$$

Therefore, for all sufficiently large $n$,

$$
E N_{n}\left(\frac{1+\epsilon}{\sqrt{\sigma}}, \frac{1+\eta}{\sqrt{\sigma}}\right) \sim \frac{1}{2 \pi} \log n .
$$

Finally, letting $(1+\eta) / \sqrt{\sigma} \leq x \leq 1$, from (2.16) and (2.17), we can show that

$$
\frac{\Delta}{A^{2}} \leq L_{0} \frac{\sqrt{\sigma}}{\sigma x^{2}-1}
$$

where the $L$ 's here and in the following are absolute constants not necessarily all possessing the same values. This together with (1.3) gives

$$
\begin{aligned}
E N_{n}\left(\frac{1+\eta}{\sqrt{\sigma}}, 1\right)= & \left(L_{0} /(2 \pi)\right)(\log n)^{1 / 3}+\left(L_{0} /(2 \pi)\right) \log \left(\frac{\sqrt{\sigma}-1}{\sqrt{\sigma}+1}\right) \\
& +\left(L_{0} /(2 \pi)\right) \log (2+\eta)=O(\log n)^{1 / 3} .
\end{aligned}
$$

Hence from (2.9), (2.11), (2.13), (2.14), (2.18) and (2.19), we have the proof of Theorem 1 for $n$ odd. For $n$ even the proof is very similar. The only difference is that an additional $\sigma$ will appear in the denominator of the right-hand side of $\Delta^{2}$ and $A^{2}$ in (2.16) and (2.17). This would not affect the analysis, and therefore the proof remains valid for $n$ even as well.

Remark 1. If $\sigma=f(n)$ such that $f(n) \rightarrow \infty$ as $n \rightarrow \infty$, the result of Theorem 1 will still hold.

In this case, from (1.3), (2.12) and (2.8), we can easily show that for $(1-\epsilon) / \sqrt{\sigma} \leq$ $x \leq(1+\epsilon) / \sqrt{\sigma}$,

$$
E N_{n}\left(\frac{1-\epsilon}{\sqrt{\sigma}}, \frac{1+\epsilon}{\sqrt{\sigma}}\right) \leq \frac{1}{\pi} \frac{2 n \epsilon}{\sqrt{f(n)}}=o(\log n) .
$$

From (2.16) and (2.17), and for $(1+\eta) / \sqrt{\sigma} \leq x \leq 1$, we obtain

$$
\frac{\Delta}{A^{2}} \leq \operatorname{Max}\left\{L_{1} \frac{\sqrt{\sigma}}{\sigma x^{2}-1}, L_{2}\right\} .
$$

The first term inside the maximum occurs at the beginning of the interval $[(1+\eta) / \sqrt{\sigma}, 1]$, and the second term occurs at the end of this interval. Therefore, (2.19) holds in this case as well. 


\section{Proof of Theorem 2}

From the assumptions of Theorem 2 for the distributions of the coefficients of $P_{n}(x)$, from (1.2), we can easily show that

$$
\begin{aligned}
A^{2} & =n \sum_{j=0}^{n-1} j x^{2 j}-\sum_{j=0}^{n-1} j^{2} x^{2 j}, \\
B^{2} & =n \sum_{j=0}^{n-1} j^{3} x^{2 j-2}-\sum_{j=0}^{n-1} j^{4} x^{2 j-2},
\end{aligned}
$$

and

$$
C=n \sum_{j=0}^{n-1} j^{2} x^{2 j-1}-\sum_{j=0}^{n-1} j^{3} x^{2 j-1} .
$$

Using (3.1)-(3.3) and a similar technique used in the proof of Theorem 1 to obtain (2.4) $-(2.6)$ will yield

$$
\begin{aligned}
A^{2}= & \frac{n x^{2}\left(1+x^{2 n}\right)}{\left(1-x^{2}\right)^{2}}-\frac{x^{2}\left(1+x^{2}\right)\left(1-x^{2 n}\right)}{\left(1-x^{2}\right)^{3}} \\
B^{2}= & \frac{n^{3} x^{2 n}\left(1-x^{2}\right)^{3}+n\left(1+3 x^{2 n}\right)\left(1+4 x^{2}+x^{4}\right)\left(1-x^{2}\right)}{\left(1-x^{2}\right)^{5}} \\
& +\frac{3 n^{2} x^{2 n}\left(1+x^{2}\right)\left(1-x^{2}\right)^{2}-\left(1-x^{2 n}\right)\left(1+11 x^{2}+11 x^{4}+x^{6}\right)}{\left(1-x^{2}\right)^{5}}
\end{aligned}
$$

and

$$
\begin{aligned}
C= & \frac{n^{2} x^{2 n+1}\left(1-x^{2}\right)^{2}+n x\left(1+2 x^{2 n}\right)\left(1+x^{2}\right)\left(1-x^{2}\right)}{\left(1-x^{2}\right)^{4}} \\
& -\frac{x\left(1-x^{2 n}\right)\left(1+4 x^{2}+x^{4}\right)}{\left(1-x^{2}\right)^{4}}
\end{aligned}
$$

Therefore, from (1.2) and (3.1)-(3.6) we obtain

$$
\begin{aligned}
\Delta^{2}= & \frac{n^{4} x^{2 n+2}\left(1-x^{2}\right)^{2}+n^{2}\left(2 x^{4}+2 x^{4 n+4}+6 x^{2 n+4}-2 x^{2 n+6}-2 x^{2 n+2}\right)}{\left(1-x^{2}\right)^{6}} \\
& +\frac{4 x^{4}\left(1+x^{2}+x^{4}\right)\left(1-x^{2 n}\right)^{2}-6 n x^{4}\left(1+x^{2}\right)\left(1-x^{2 n}\right)\left(1-x^{2}\right)}{\left(1-x^{2}\right)^{8}}
\end{aligned}
$$

When $0 \leq x \leq 1-\epsilon$, where $\epsilon$ is given in (2.8), and for all sufficiently large $n$, we can easily show that

$$
x^{n} \leq n^{-10} .
$$

Therefore, from (3.4) and (3.7), we have

$$
\begin{aligned}
\Delta^{2}= & \frac{n^{2} x^{4}}{\left(1-x^{2}\right)^{6}}\left[2-\frac{6\left(1+x^{2}\right)}{n\left(1-x^{2}\right)}+\frac{4\left(1+x^{2}+x^{4}\right)}{n^{2}\left(1-x^{2}\right)^{2}}+O\left(n^{-20}\right)\right] \\
= & \frac{n^{2} x^{4}}{\left(1-x^{2}\right)^{6}}\left[2+O\left((n \epsilon)^{-1}\right)\right]
\end{aligned}
$$


and

$$
\begin{aligned}
A^{2}= & \frac{n x^{2}}{\left(1-x^{2}\right)^{2}}\left[1-\frac{1+x^{2}}{n\left(1-x^{2}\right)}+O\left(n^{-22}\right)\right] \\
= & \frac{n x^{2}}{\left(1-x^{2}\right)^{2}}\left[1+O\left((n \epsilon)^{-1}\right)\right] .
\end{aligned}
$$

Hence, from (3.9) and (3.8), we can easily show that for $n$ sufficiently large,

$$
\frac{\Delta}{A^{2}} \sim \frac{\sqrt{2}}{1-x^{2}}
$$

Therefore, from (1.3), (2.8) and (3.10), we obtain

$$
E N_{n}(0,1-\epsilon) \sim \frac{\sqrt{2}}{2 \pi} \log n .
$$

Now let $1-\epsilon \leq x \leq 1-\delta$, where $\delta$ is defined in (2.10). From (3.4) and (3.7), we can show that

$$
\begin{aligned}
\Delta^{2}= & \left(\frac{n^{4} x^{4}}{\left(1-x^{2}\right)^{4}}\right)\left[x^{2 n-2}+\frac{2+2 x^{4 n}+6 x^{2 n}-2 x^{2 n+2}-2 x^{2 n-2}}{n^{2}\left(1-x^{2}\right)^{2}}\right. \\
& \left.-\frac{6\left(1+x^{2}\right)\left(1-x^{2 n}\right)}{n^{3}\left(1-x^{2}\right)^{3}}+\frac{4\left(1+x^{2}+x^{4}\right)\left(1-x^{2 n}\right)^{2}}{n^{4}\left(1-x^{2}\right)^{4}}\right]
\end{aligned}
$$

and

(3.13) $A^{2}=\left(\frac{n x^{2}}{\left(1-x^{2}\right)^{2}}\right)\left[1+x^{2 n}-\frac{\left(1-x^{2 n}\right)}{n\left(1-x^{2}\right)}\right]$.

Since in this interval, for all $n$ sufficiently large, $x^{n} \leq \exp (-\log \log n)$ and $n\left(1-x^{2}\right)=O(\log \log n)$, from (3.12) and (3.13), we have

$$
\frac{\Delta}{A^{2}}=O\left(\frac{n}{\log \log n}\right) \text {. }
$$

Therefore, from (1.3), (2.8), (2.10) and (3.14), we obtain

$$
E N_{n}(1-\epsilon, 1-\delta)=o(\log n) \text {. }
$$

Finally, letting $1-\delta \leq x \leq 1$, from (1.2) and (2.12), we have

$$
\frac{\Delta}{A^{2}} \leq n \text {. }
$$

This together with (1.3) shows that

$$
E N_{n}(1-\delta, 1)=O(\log \log n) .
$$

Hence from (3.11), (3.15) and (3.16), we have the proof of Theorem 2.

\section{REFERENCES}

[1] A.T. Bharucha-Reid and M. Sambandham. Random Polynomials. Academic Press, New York, 1986. MR:87m:60118

[2] A. Edelman and E. Kostlan. How many zeros of a random polynomial are real? Bull. Amer. Math. Soc., 32:1-37, 1995. MR95m:60082

[3] K. Farahmand. Topics in Random Polynomials. Addison Wesley Longman, London, 1998. MR 2000d:60092

[4] K. Farahmand. On random algebraic polynomials. Proc. Amer. Math. Soc., 127:3339-3344, 1999. MR2000b:60130

[5] K. Farahmand and P. Hannigan. The expected number of local maxima of a random algebraic polynomial. J. Theoretical Probability, 10:991-1002, 1997. MR 99a:60053 
[6] K. Farahmand and P. Hannigan. Large level crossings of random polynomials. Stochastic Analysis and Applications, 20:299-309, 2002. MR2003d:60107

[7] K. Farahmand and M. Sambandham. Real zeros of classes of random algebraic polynomials. J. Appl. Math. and Stochastic Analysis, 16:249-255, 2003.

[8] M. Kac. On the average number of real roots of a random algebraic equation. Bull. Amer. Math. Soc., 49:314-320, 1943. MR 4:196d

[9] B.F. Logan and L.A. Shepp. Real zeros of random polynomials. Proc. London Math. Soc., 18:29-35, 1968. MR.38:2829

[10] B.F. Logan and L.A. Shepp. Real zeros of random polynomials. II. Proc. London Math. Soc., 18:308-314, 1968. MR38:2830

[11] S.O. Rice. Mathematical theory of random noise. Bell System Tech. J., 25:46-156, 1945. Reprinted in: Selected Papers on Noise and Stochastic Processes (ed. N. Wax), Dover, New York, 1954, 133-294. MR6:233i

[12] M. Sambandham. On a random algebraic polynomial. J. Indian Math. Soc., 41:83-97, 1977. MR:58:31391

[13] M. Sambandham. On the average number of real zeros of a class of random algebraic curves. Pacific J. Math., 81:207-215, 1979. MR80h:60055

[14] J.E. Wilkins. An asymptotic expansion for the expected number of real zeros of a random polynomial. Proc. Amer. Math. Soc., 103:1249-1258, 1988. MR90f:60105

Department of Mathematics, University of Ulster at Jordanstown, Co. Antrim, BT37 0QB, United Kingdom

E-mail address: K.Farahmand@ulst.ac.uk

Department of Statistics, School of Mathematical Sciences, Ferdowsi University of Mashhad, P.O. Box 1159-91775, MashHan, Iran

E-mail address: Nezakati@math.um.ac.ir 